\title{
Prevalence and risk factors of non-alcoholic fatty liver disease in southwest Iran: a population-based case-control study
}

\author{
Elaheh Zarean', Reza Goujani', Ghorbanali Rahimian², Ali Ahamdi³ \\ 'Modeling in Health Research Center, Shahrekord University of Medical Sciences, Shahrekord, Iran \\ 2Department of Gastroenterology and Hepatology, School of Medicine, Shahrekord University of Medical Sciences, Shahrekord, Iran \\ ${ }^{3}$ Modeling in Health Research Center and School of Public Health, Department of Epidemiology and Biostatistics, Shahrekord University \\ of Medical Sciences, Shahrekord, Iran
}

\begin{abstract}
Aim of the study: Few population-based studies have been conducted to report the prevalence of non-alcoholic fatty liver disease (NAFLD) and determine its risk factors in Iran, particularly in Shahrekord, southwest Iran. The main aim of this population-based, case-control study was to determine the prevalence of NAFLD and its potential risk factors in an Iranian population-based cohort study in Shahrekord, Iran.

Material and methods: We compared 1153 cases with NAFLD at 35 to 70 years to 1153 frequency-matched NAFLD-free controls from a population-based Shahrekord cohort study. Data collection and assessed risk factors (demographics, anthropometrics, clinical and biochemical factors, and laboratory tests) were performed based on the Shahrekord cohort study protocol. Chi-square test, stepwise selection with backward likelihood ratio, logistic regression and log-binomial regression model with 95\% confidence interval were performed to determine risk factors with significant effects on NAFLD.

Results: In this study, the mean ( \pm standard deviation) age of the participants was $50.23( \pm 8.70)$ years. The prevalence of NAFLD and metabolic syndrome with 95\% confidence interval were $16.47 \%$ (15.61-17.36\%) and $32.74 \%(30.83-34.70 \%)$, respectively. There was a significant difference in the frequency of waist circumstance, triglycerides, body mass index, blood pressure, and alanine aminotransferase between cases and controls $(p<0.001)$. According to log-binomial model results, socioeconomic status, hypertension, diabetes, and physical activity were significantly associated with NAFLD.

Conclusions: Our findings have important clinical and public health implications in southwest Iran for monitoring the prevalence of NAFLD and important risk factors to manage, screen, prevent, and reduce NAFLD and related factors.
\end{abstract}

Key words: risk factor, prevalence, non-alcoholic fatty liver disease, Shahrekord cohort study, log-binomial regression model.

\section{Address for correspondence}

Dr. Ali Ahamdi, Modeling in Health Research Center and School of Public Health, Department of Epidemiology and Biostatistics, Shahrekord University of Medical Sciences, Shahrekord, Iran, e-mail: aliahmadi2007@gmail.com

\section{Introduction}

Non-alcoholic fatty liver disease (NAFLD) is one of the significant types of liver diseases in patients with metabolic comorbidities, such as obesity, diabetes mellitus, and dyslipidemia [1]. According to practice guidance from the American Association for the Study of Liver Disease, NAFLD is defined as the presence of hepatic steatosis (HS) in absence of secondary causes of hepatic fat accumulation including significant alcohol intake, use of a steatogenic medication for a long time, or monogenic hereditary disorders $[1,2]$. With 
regards to etiological criteria, NAFLD is classified as non-alcoholic fatty liver (NAFL) and non-alcoholic steatohepatitis (NASH) with or without cirrhosis [3]. NAFL is defined as the presence of over $5 \%$ of HS, and lack of hepatocellular injury in the form of hepatocyte ballooning [1].

Due to the increasing prevalence of obesity and changes in lifestyle, for example, variation in dietary habits along with decreased physical activity in the eastern countries, the prevalence of NAFLD dramatically increased in the previous decade $[4,5]$. At present, various studies and guidelines demonstrate that there is a close association between NAFLD and metabolic risk factors, particularly dyslipidemia, type 2 diabetes mellitus (T2DM) and visceral obesity and other components of metabolic syndrome (METS) [4]. While all metabolic disorders are known as risk factors for NAFLD, the high prevalence of NAFLD is predictable [5]. However, according to the diagnostic tools used, the reported prevalence of NAFLD is $10-35 \%$ in the general population [4]. There is important variation in the prevalence and risk factors for NAFLD in the Iranian population among different geographical regions [4]. According to several epidemiological studies in Iran, T2DM is highly associated with NAFLD. Moreover, NAFLD and NASH prevalence in Iranian patients with T2DM is $55.8 \%$ [6], and has changed from $2.9 \%$ to $7.1 \%$ in the general population [7-9]. Another important risk factor of NAFLD, which is defined by income and education, was socioeconomic status [10]. Interestingly, medical researchers have shown that fatty liver is also associated with hypertension [11]. In particular, NAFLD was found to occur more frequently in hypertensive patients [11].

Because few studies have been conducted on the prevalence of NAFLD in southwest Iran, particularly Shahrekord (height above mean sea level is around $2153 \mathrm{~m}$ and therefore this city is known as Iran's roof), monitoring the prevalence of NAFLD and important risk factors is essential for management, screening, prevention, and reduction of NAFLD and related factors. Several studies have used regression analysis to identify and determine risk factors for NAFLD as a dichotomous outcome [5]. Due to increasing use of multiple logistic regression for modeling dichotomous outcomes in medical research, the need for efficient models with greater accuracy has been intensified in certain situations [12]. In epidemiological studies, the dichotomous outcome is frequently analyzed by logistic regression. Since the prevalence ratio is overestimated by the odds ratio in logistic regression, a log-binomial regression model can provide correct estimates and serve as a better alternative than logistic regression [13].
The main aim of this population-based, casecontrol study was to determine the prevalence of NAFLD and its potential risk factors in an Iranian population-based cohort study in Shahrekord, southwest Iran.

\section{Material and methods}

\section{Study type}

This population-based, case-control study was conducted within the Shahrekord Cohort Study (SCS) on subjects aged 35-70 years. This study was designed to serve as one of the centers of the Prospective Epidemiological Research Studies in Iran (PERSIAN) Cohort and is being conducted in Chaharmahal and Bakhtiari province, southwest of Iran. First, NAFLD prevalence in the whole cohort was estimated. Then, the individuals with NAFLD $(n=1153)$ were considered cases. 1153 controls were selected from sex-frequency matched subjects without NAFLD in the SCS. The sample size of the original cohort was 10078 people, with a 20 -year follow-up, who were selected by a multistage, stratified cluster random sampling. The study protocol of the SCS was reviewed and approved by the Ethics Committee of Shahrekord University of Medical Sciences (IR.SKUMS.REC 1394.286). Details of the SCS protocol have been published in other studies [14, 15].

\section{Case and control population, the inclusion and exclusion criteria, risk factors}

Patients with chronic liver disease, patients with a history of significant excessive alcohol consumption (more than $20 \mathrm{~g}$ daily for women and $30 \mathrm{~g}$ daily for men) who were regarded as suffering from alcoholic liver disease [16], patients with viral and autoimmune hepatitis, and patients with cognitive disorders and women who were pregnant were excluded from study. To participate in the SCS, people were invited by a dated letter of invitation given to them in person and then, before the day of their referral, they were recalled by telephone [14].

Data on demographics, socioeconomic status and anthropometric characteristics, occupational history and exposures, lifestyle and physical activity were collected using standard questionnaires. Physical examinations and medical questionnaire were also used to investigate history of chronic diseases, recommended pharmacological therapies for NAFLD/NASH based on guidelines (metformin hydrochloride, pioglitazone, vitamin E, ursodeoxycholic acid) [17], family medical history and individual habits (smoking and alcohol use). A standard barometer was used to measure blood pressure in 
the right and left arms twice (with a 15-min interval); finally, the average of the two measures of the left and right arm was considered the blood pressure. Biochemical tests including fasting blood sugar, serum cholesterol, triglycerides, aspartate aminotransferase (AST), alanine aminotransferase (ALT), high-density lipoprotein (HDL), low-density lipoprotein (LDL) and cholesterol were measured by using blood samples of participants with a Pars Azmoon kit [14]. NAFLD was diagnosed by a hepatologist on the basis of ultrasonography and medical records. The definition of National Cholesterol Education Program Adult Treatment Panel III criteria (ATPIII) was used for diagnosis of METS [18]. The presence of any 3 of the following 5 criteria was required for the diagnosis: fasting serum glucose $\geq 100 \mathrm{mg} / \mathrm{dl}$, waist circumferences (WC) ( $>102 \mathrm{~cm}$ in men; $>88 \mathrm{~cm}$ in women), triglyceride (TG) $\geq 150 \mathrm{mg} / \mathrm{dl}$, HDL ( $<40 \mathrm{mg} / \mathrm{dl}$ in men; $<50 \mathrm{mg} / \mathrm{dl}$ in women), blood pressure (BP) $\geq 140$ (SBP) or $\geq 90$ (DBP) $\mathrm{mmHg}$.

Additionally, socioeconomic status was determined by measuring a combination of education level and a checklist of income and occupation. Principle component factor analysis (KMO $=0.719$, Bartlett's sphericity test and $p$-value $<0.001)$ was applied to determine the socioeconomic status. The socioeconomic status factor was converted to an ordinal variable and was labeled as low, medium, and high level.

\section{Statistical analysis}

Demographics and historical exposure were categorized according to the SCS protocol and METS definition. Descriptive characteristics of the participant were expressed as the mean \pm standard deviation (SD) and frequency (percentage) for continuous and categorical variables, respectively.

Two separate frequency tables described all variables in the total population including prevalence of NAFLD in each category of variables for METS as well as demographic and exposure variables, respectively. The frequency of each pharmacological therapy which was taken by patients with NAFLD was reported.

The $\chi^{2}$ test was applied to compare the frequency of these variables between the patients who had NAFLD and controls who did not have NAFLD. Stepwise selection with backward likelihood ratio was used to investigate the best subset of variables in which the best fit in the logistic regression model could be achieved. Variance inflation factor (VIF) was used to investigate the presence of multicollinearity between the covariates. The best subset of variables which were drawn from the logistic regression model was assessed in the log-binomial regression fitting. Finally, a log-binomial regression model was used to determine risk factors that had significant effects on NAFLD. The results were presented as risk ratios (RRs) from the log-binomial regression model. The RR in the log-binomial model demonstrates in which groups of variables the risk of interested outcome is greater than that in the other groups [19]. If the value of RR in the log-binomial model is greater than one, the risk of the outcome in the baseline group is more than that in the other group and vice versa [20]. The 95\% confidence intervals (95\% CI) were demonstrated for the estimated effect sizes. Stata software, version 13 , was used to perform the models and a $p$-value $<0.05$ was considered the level of statistical significance.

\section{Results}

The mean ( \pm standard deviation) age was 50.23 $( \pm 8.70)$ years. The prevalence $(95 \% \mathrm{CI})$ of NAFLD and METS in this study was $16.47 \%$ (15.61-17.36\%) and $32.74 \%$ (30.83-34.70\%), respectively. Mean (and standard deviation) body mass index (BMI) in case and control groups was 30.02 (4.48) and 27.88 (4.39), respectively.

The participants' characteristics and $\chi^{2}$ test results are shown in Table 1 and Table 2. According to the results of Table 1 and Table 2, the difference in prevalence between the patients who had NAFLD and the controls who did not have NAFLD was significant with respect to diabetes $(p<0.001)$, hypertension $(p<0.001)$, physical activity $(p<0.001)$, and METS $(p<0.001)$. Furthermore, the results of Table 2 show that there is a significant difference between the frequency of WC, TG, BMI, BP and ALT (all p-values $<0.001)$ between cases and controls. Three components of metabolic syndrome which were not significantly different between the two groups were fasting serum glucose, HDL and total cholesterol (Table 2). The stepwise selection indicated that socioeconomic status, hypertension, alcohol consumption, diabetes, physical activity, and METS had the best fit in the logistic model while the presence of multicollinearity between covariates was investigated based on the VIF values. Therefore, all these variables were used in the log-binomial regression model.

The results of the log-binomial regression model are presented in Table 3. According to the estimations of the log-binomial model, socioeconomic status, hypertension, diabetes and physical activity were significantly associated with NAFLD. Subjects who had a low/medium level of socioeconomic status were less likely to have NAFLD than individuals who had a high socioeconomic level (low level: $\mathrm{RR}=0.88,95 \%$ CI: 0.80-0.96, $p=0.004$; 
Table 1. Frequency of demographic characteristics and potential risk factors of participants by NAFLD status along with chi-square test

\begin{tabular}{|c|c|c|c|c|}
\hline \multirow[t]{2}{*}{ Variable } & \multirow[t]{2}{*}{ Total (\%) } & \multicolumn{2}{|c|}{ NAFLD status } & \multirow[t]{2}{*}{$P$-value } \\
\hline & & Yes $(\%)$ & № (\%) & \\
\hline Age (years) & & & & 0.459 \\
\hline$<40$ & $289(12.5)$ & $135(46.7)$ & $154(53.3)$ & \\
\hline $40-60$ & $1631(70.7)$ & $820(50.3)$ & $811(49.7)$ & \\
\hline$\geqslant 60$ & $386(16.7)$ & $198(51.3)$ & $188(48.7)$ & \\
\hline Gender & & & & 0.994 \\
\hline Male & $920(39.9)$ & $460(50.0)$ & $460(50.0)$ & \\
\hline Female & $1386(60.1)$ & $693(50.0)$ & $693(50.0)$ & \\
\hline Education & & & & 0.419 \\
\hline$\leqslant$ Diploma degree & $1584(68.7)$ & $783(49.4)$ & $801(50.6)$ & \\
\hline > Diploma degree & $722(31.3)$ & $370(51.2)$ & $352(48.8)$ & \\
\hline Marital status & & & & 0.115 \\
\hline Single & $33(1.4)$ & $12(36.4)$ & $21(63.6)$ & \\
\hline Married & $2170(94.1)$ & $1096(50.5)$ & $1074(49.5)$ & \\
\hline Widowed/Divorced & $103(4.5)$ & $45(43.7)$ & $58(56.3)$ & \\
\hline Ethnicity & & & & 0.072 \\
\hline Fars & $1398(60.6)$ & $679(48.6)$ & $719(51.4)$ & \\
\hline Tork & $279(12.1)$ & $135(48.4)$ & $144(51.6)$ & \\
\hline Lor & $629(27.3)$ & $339(53.9)$ & $290(50)$ & \\
\hline Job status & & & & 0.676 \\
\hline Unemployment & $1248(54.1)$ & $629(50.4)$ & $619(49.6)$ & \\
\hline Employment & $1058(45.9)$ & $524(49.5)$ & $534(50.5)$ & \\
\hline Socioeconomic status & & & & 0.112 \\
\hline Low level & $710(30.8)$ & $337(47.5)$ & $373(52.5)$ & \\
\hline Medium level & $929(40.3)$ & $467(49.7)$ & $467(50.3)$ & \\
\hline High level & $667(28.9)$ & $313(53.1)$ & $313(46.9)$ & \\
\hline Alcohol consumption & & & & 0.335 \\
\hline Yes & 371 (16.1) & $194(52.3)$ & $177(47.7)$ & \\
\hline No & $1935(83.9)$ & $959(49.6)$ & $976(50.4)$ & \\
\hline Type 2 diabetes mellitus & & & & $<0.001$ \\
\hline Yes & $329(16.1)$ & $229(69.6)$ & $100(30.4)$ & \\
\hline No & $1977(83.9)$ & $924(46.7)$ & $1053(53.3)$ & \\
\hline Hypertension (SBP $\geqslant 140$ or $\mathrm{DBP} \geqslant 90 \mathrm{mmHg}$ ) & & & & $<0.001$ \\
\hline Yes & $523(22.7)$ & $332(63.5)$ & $191(36.5)$ & \\
\hline No & $1783(77.3)$ & $821(46.0)$ & $962(54.0)$ & \\
\hline Ischemic heart disease & & & & 0.133 \\
\hline Yes & $154(6.7)$ & $86(55.8)$ & $68(44.2)$ & \\
\hline No & $2152(93.3)$ & $1067(49.6)$ & $1085(50.4)$ & \\
\hline Smoking & & & & 0.853 \\
\hline Yes & $301(13.1)$ & $152(50.5)$ & $149(49.5)$ & \\
\hline No & 2005 (86.9) & $1001(49.9)$ & $1004(50.1)$ & \\
\hline Physical activity & & & & $<0.001$ \\
\hline Very low & $223(9.7)$ & $135(60.5)$ & $88(39.5)$ & \\
\hline Low & $1125(48.8)$ & $590(52.4)$ & $535(47.6)$ & \\
\hline Moderate & $722(31.3)$ & $344(47.6)$ & $378(52.4)$ & \\
\hline High & $236(10.2)$ & 84 (35.6) & $152(64.4)$ & \\
\hline
\end{tabular}


medium level: $\mathrm{RR}=0.80,95 \%$ CI: 0.72-0.88, $p<0.001$ ). In the current study people with T2DM had 1.34 times higher risk of having NAFLD than people who did not have T2DM $(\mathrm{RR}=1.34,95 \% \mathrm{CI}: 1.22-1.47, p<0.001)$.
The risk of having NAFLD in participants with hypertension was significantly higher than in those without hypertension $(\mathrm{RR}=1.19,95 \% \mathrm{CI}: 1.08-1.31, p<0.001)$. In participants who had very low, low, and moderate physi-

Table 2. Prevalence of NAFLD in biochemical parameters, anthropometric indexes, and metabolic syndrome components according to NAFLD status along with the results of chi-square test

\begin{tabular}{|c|c|c|c|c|}
\hline \multirow[t]{2}{*}{ Variable } & \multirow[t]{2}{*}{$n(\%)$} & \multicolumn{2}{|c|}{ NAFLD status } & \multirow[t]{2}{*}{$P$-value } \\
\hline & & Yes (\%) & No (\%) & \\
\hline Waist circumference (cm) & & & & $<0.001$ \\
\hline$>102$ in males & $221(15.9)$ & $147(66.5)$ & $74(33.5)$ & \\
\hline$>88$ in females & $1173(84.1)$ & $590(50.3)$ & $583(49.7)$ & \\
\hline Blood pressure (mmHg) & & & & $<0.001$ \\
\hline $\mathrm{SBP} \geqslant 140$ & $354(15.4)$ & $184(52)$ & $170(48)$ & \\
\hline $\mathrm{DBP} \geqslant 90$ & $507(22)$ & $273(53.8)$ & $234(46.2)$ & \\
\hline Fasting serum glucose $(\mathrm{mg} / \mathrm{dl}) \geqslant 100$ & $1080(46.8)$ & $524(48.5)$ & $556(51.5)$ & 0.182 \\
\hline Triglycerides $(\mathrm{mg} / \mathrm{dl}) \geqslant 150$ & $1199(52.0)$ & $685(57.1)$ & $514(42.9)$ & $<0.001$ \\
\hline $\mathrm{HDL}(\mathrm{mg} / \mathrm{dl})$ & & & & 0.609 \\
\hline$<40$ in males & $168(18.3)$ & $87(51.8)$ & $81(48.2)$ & \\
\hline$<50$ in females & $425(30.7)$ & $222(52.2)$ & $203(47.8)$ & \\
\hline Serum cholesterol $(\mathrm{mg} / \mathrm{dl})>200$ & $927(40.2)$ & $450(48.5)$ & $477(5105)$ & 0.251 \\
\hline $\operatorname{ALT}(\mathrm{U} / \mathrm{I}) \geqslant 40$ & $258(11.2)$ & $170(65.9)$ & $88(34.1)$ & $<0.001$ \\
\hline AST $(U / I) \geqslant 40$ & $54(2.3)$ & $33(61.1)$ & $21(38.9)$ & 0.098 \\
\hline $\mathrm{BMI}\left(\mathrm{kg} / \mathrm{m}^{2}\right)$ & & & & $<0.001$ \\
\hline$<25$ (normal) & $397(17.2)$ & $115(29.0)$ & $282(71.0)$ & \\
\hline$\geqslant 25-<30$ (overweight) & $1077(46.7)$ & $529(41.9)$ & $548(50.9)$ & \\
\hline$\geqslant 30$ (obese) & $832(36.1)$ & $509(61.2)$ & $323(38.8)$ & \\
\hline Metabolic syndrome (ATPIII) & & & & $<0.001$ \\
\hline Yes & $775(33.6)$ & $431(55.6)$ & $344(44.4)$ & \\
\hline No & $1531(66.4)$ & $722(47.2)$ & $809(52.8)$ & \\
\hline
\end{tabular}

Table 3. Estimation based on log-binomial regression model using best subset variables according to stepwise selection (backward LR)

\begin{tabular}{|c|c|c|c|c|c|}
\hline \multirow[t]{2}{*}{ Variable } & \multirow[t]{2}{*}{ Risk ratio } & \multirow[t]{2}{*}{ Standard error } & \multicolumn{2}{|c|}{ 95\% confidence interval } & \multirow[t]{2}{*}{$P$-value } \\
\hline & & & Lower & Upper & \\
\hline \multicolumn{6}{|l|}{ Socioeconomic status } \\
\hline Medium level & 0.80 & 0.04 & 0.72 & 0.88 & $<0.001$ \\
\hline Low level & 0.88 & 0.04 & 0.80 & 0.96 & 0.004 \\
\hline \multicolumn{6}{|l|}{ Physical activity } \\
\hline Moderate & 1.26 & 0.12 & 1.05 & 1.52 & 0.012 \\
\hline Low & 1.38 & 0.12 & 1.16 & 1.65 & $<0.001$ \\
\hline Very low & 1.46 & 0.15 & 1.20 & 1.78 & $<0.001$ \\
\hline Type 2 diabetes (yes) & 1.34 & 0.06 & 1.22 & 1.47 & $<0.001$ \\
\hline Hypertension (yes) & 1.19 & 0.06 & 1.08 & 1.31 & $<0.001$ \\
\hline Alcohol consumption (yes) & 1.11 & 0.06 & 1.00 & 1.23 & 0.058 \\
\hline METS (yes) & 1.08 & 0.05 & 0.98 & 1.19 & 0.102 \\
\hline
\end{tabular}


cal activity the risk of having NAFLD was $1.46,1.38$, and 1.26 times higher than in those who had a high level of physical activity (very low level: $\mathrm{RR}=1.46,95 \% \mathrm{CI}: 1.20$ $1.78, p<0.001$; low level: $\mathrm{RR}=1.38,95 \%$ CI: 1.16-1.65, $p<0.001$; moderate level: $\mathrm{RR}=1.26,95 \%$ CI: 1.05-1.52, $p=0.012$ ). Alcohol consumption was not identified as a significant factor in the log-binomial model. However, the risk of having NAFLD in patient who used alcohol was 1.11 times higher than in those who did not consume alcohol ( $\mathrm{RR}=1.11,95 \%$ CI: $1.00-1.23, p=0.058)$.

A list of recommended pharmacological therapies for NAFLD based on guidelines was provided. Our results indicated that the frequency and the percentage of using metformin hydrochloride among NAFLD patients are higher than for other drugs [metformin hydrochloride: 248 (6.42); pioglitazone: 4 (0.103); vitamin E: 26 (0.67); ursodeoxycholic acid: 5 (0.12)].

\section{Discussion}

In the current study, we investigated the prevalence of NAFLD and its associated risk factors in an Iranian population at enrolment phases of the SCS. Our results showed that the prevalence of NAFLD was $16.47 \%$, and after adjusting for confounding variables type 2 diabetes mellitus, hypertension, socioeconomic status and physical activity were significantly associated with NAFLD. To the best of our knowledge, this is the first study to report the prevalence of NAFLD and establish its risk factors in a relatively large number of subjects in southwest Iran.

According to recent epidemiological studies worldwide, the global prevalence of NAFLD has been reported at 24\% [21]. Although NAFLD is regarded as a prevalent disease of high burden in all countries, the highest rate belongs to the Middle East (32\%) and South America (31\%) [21]. As expected, there is wide variation in prevalence of NAFLD in the Asia-Pacific region associated with disparities in socioeconomic level as well as lifestyle differences among countries of the region [22]. Consistent with these findings, there was an obvious difference in prevalence of NAFLD between the current study and the other ones conducted in the Asia-Pacific region. This notable variation may be attributed to the type of diagnostic tools used in our study and also the absence of misclassification in diagnosis of NAFLD. Since in our study the criteria for NAFLD diagnosis were both the confirmation of NAFLD by a hepatologist and liver sonography, individuals who did not fulfill these criteria were not included in this study. Furthermore, because of the benign and reversible condition of NAFLD and absence of obvious and specific clinical symptoms, diagnosis of
NAFLD can be a complex process. Therefore, NAFLD may also be observed in non-obese individuals, patients without clinical manifestations of METS, and all people who do not refer for diagnostic tests. These reasons may lead to a wide variation in the prevalence of NAFLD (10-35\%) in population-based studies [4, 23].

In our study, a borderline significant association between alcohol consumption and NAFLD was found in the log-binomial model. Various studies have demonstrated that low-moderate alcohol consumption might decrease the likelihood of developing NAFLD [24] and excessive use of alcohol is the cause of most cases of fatty liver disease, but recently, diverse factors such as insulin resistance, oxidative stress, mitochondrial dysfunction, immune deregulation, and adipokines have become more important in the pathogenesis of NAFLD [25].

Several studies especially in Iran have shown that NAFLD is associated with METS [5]. However, in the current study, the effect size of METS was not significantly associated with NAFLD. Explicit epidemiological evidence has shown that all NAFLD patients may not be classified as cases of METS, and also all patients with METS may not develop NAFLD. Therefore, there are some other risk factors that are not related to METS yet cause NAFLD in patients [26].

Studies recently conducted in the US have demonstrated that T2DM and NAFLD have common risk factors and there is a strong association between them [27]. T2DM was also found to be one of the risk factors for NAFLD in the current study. Although T2DM appears to intensify the progress of NAFLD, the liver disease makes diabetes management more problematic and the mechanism of this association remains unknown. Diabetes may encourage the development of NASH, i.e., a more severe form of the disease, and increase the risk of cirrhosis and hepatocellular carcinoma [8].

The association between NAFLD and hypertension has been a controversial issue in several studies. Many clinical researchers have evaluated the relationship between these two diseases with or without other accompanying factors [28]. Our findings are in accordance with recent studies, and hypertension is a risk factor for NAFLD. A probable mechanism of this association is that hypertension has a significant effect in the pathogenesis of METS, which in turn can influence the progression of NAFLD in patients with hypertension [28].

Interestingly, physical activity has been considered a vital factor for managing NAFLD in several studies. These studies have discussed the beneficial effect of increasing physical activity on reducing the progress of NAFLD, and also they have argued that promoting 
and maintaining physical activity provide health benefits for NAFLD patients [29]. In our study, a strong association was found between NAFLD and very low, low and moderate levels of physical activity.

In the current study, although socioeconomic status was one of the most significant contributing factors associated with NAFLD, patients in low and moderate socioeconomic classes had lower risk of developing NAFLD. These findings have also been obtained in several studies such as those of Santos et al. and Manuck et al. [30, 31].

Our study had some limitation regarding NAFLD diagnosis. Although ultrasonography and medical records were used for diagnosis of NAFLD in this study, liver biopsy could have also been a gold standard method for NAFLD diagnosis. However, because of research ethics, we did not use it. A large sample size which can be representative of the study population, high-quality data, proper statistical method and also controlling for confounding variables are the most important advantages of this study.

\section{Conclusions}

Our findings have important clinical and public health implications in southwest Iran for monitoring the prevalence of NAFLD. The current study, as a clinical report, established 4 risk factors (hypertension, type 2 diabetes mellitus, physical activity, and socioeconomic status) for NAFLD. This study suggests that these significant risk factors should be taken into account in the screening, prevention, and reduction of NAFLD.

Ethical code: IR.SKUMS.REC.1397.160.

\section{Disclosure}

The authors report no conflict of interest.

\section{References}

1. Chalasani N, Younossi Z, Lavine JE, et al. The diagnosis and management of nonalcoholic fatty liver disease: practice guidance from the American Association for the Study of Liver Diseases. Hepatology 2018; 67: 328-357.

2. Younossi ZM, Koenig AB, Abdelatif D, et al. Global epidemiology of nonalcoholic fatty liver disease - meta-analytic assessment of prevalence, incidence, and outcomes. Hepatology 2016; 64: 73-84.

3. Streba LA, Vere CC, Rogoveanu I, Streba CT. Nonalcoholic fatty liver disease, metabolic risk factors, and hepatocellular carcinoma: an open question. World J Gastroenterol 2015; 21: 41034110.

4. Lankarani KB, Ghaffarpasand F, Mahmoodi M, et al. Non alcoholic fatty liver disease in southern Iran: a population based study. Hepat Mon 2013; 13: e9248.
5. Amirkalali B, Poustchi H, Keyvani H, et al. Prevalence of non-alcoholic fatty liver disease and its predictors in north of Iran. Iran J Public Health 2014; 43: 1275-1283.

6. Merat S, Yarahmadi S, Tahaghoghi S, et al. Prevalence of fatty liver disease among type 2 diabetes mellitus patients and its relation to insulin resistance. Mid East J Dig Dis (MEJDD) 2009; 1: 74-79.

7. Khoonsari M, Mohammad Hosseini Azar M, Ghavam R, et al. Clinical manifestations and diagnosis of nonalcoholic fatty liver disease. Iran J Pathol 2017; 12: 99-105.

8. Bril F, Cusi K. Management of nonalcoholic fatty liver disease in patients with type 2 diabetes: a call to action. Diabetes Care 2017; 40: 419-430.

9. Dallio M, Masarone M, Caprio GG, et al. Endocan serum levels in patients with non-alcoholic fatty liver disease with or without type 2 diabetes mellitus: a pilot study. J Gastrointestin Liver Dis 2017; 26: 261-268.

10. Jia G, Li X, Wang L, et al. Relationship of socioeconomic status and non-alcoholic fatty liver disease in patients with type 2 diabetes mellitus. Zhonghua Gan Zang Bing Za Zhi 2015; 23: 760-764.

11. Donati G, Stagni B, Piscaglia F, et al. Increased prevalence of fatty liver in arterial hypertensive patients with normal liver enzymes: role of insulin resistance. Gut 2004; 53: 1020-1023.

12. Bagley SC, White H, Golomb BA. Golomb, logistic regression in the medical literature: standards for use and reporting, with particular attention to one medical domain. J Clin Epidemiol 2001; 54: 979-985.

13. Barros AJ, Hirakata VN. Alternatives for logistic regression in cross-sectional studies: an empirical comparison of models that directly estimate the prevalence ratio. BMC Med Res Methodol 2003; 3: 21 .

14. Khaledifar A, Hashemzadeh M, Solati K, et al. The protocol of a population-based prospective cohort study in southwest of Iran to analyze common non-communicable diseases: Shahrekord cohort study. BMC Public Health 2018; 18: 660.

15. Poustchi H, Eghtesad S, Kamangar F, et al. Prospective epidemiological research studies in IrAN (The PERSIAN Cohort): rationale, objectives and design. Am J Epidemiol 2018; 187: 647-655.

16. Cao G, Yi T, Liu Q, et al. Alcohol consumption and risk of fatty liver disease: a meta-analysis. PeerJ 2016; 4: e2633-e2633.

17. Chalasani N, Younossi Z, Lavine JE, et al. The diagnosis and management of non-alcoholic fatty liver disease: practice Guideline by the American Association for the Study of Liver Diseases, American College of Gastroenterology, and the American Gastroenterological Association. Hepatology 2012; 55: 2005-2023.

18. Hua Q, Yin CI. Clinical management of metabolic syndrome. Adv Cardiovasc Dis 2006; 6: 2.

19. McNutt LA, Wu C, Xue X, Hafner JP, et al. Estimating the relative risk in cohort studies and clinical trials of common outcomes. Am J Epidemiol 2003; 157: 940-943.

20. O'Connor AM. Interpretation of odds and risk ratios. J Vet Intern Med 2013; 27: 600-603.

21. Younossi Z, Anstee QM, Marietti M, et al. Global burden of NAFLD and NASH: trends, predictions, risk factors and prevention. Nat Rev Gastroenterol Hepatol 2018; 15: 11-20.

22. Benedict M, Zhang X. Non-alcoholic fatty liver disease: An expanded review. World J Hepatol 2017; 9: 715-732.

23. Vernon G, Baranova A, Younossi ZM. Systematic review: the epidemiology and natural history of non-alcoholic fatty liver disease and non-alcoholic steatohepatitis in adults. Aliment Pharmacol Ther 2011; 34: 274-285. 
24. Cao G, Yi T, Liu Q, et al. Alcohol consumption and risk of fatty liver disease: a meta-analysis. PeerJ 2016; 4: e2633.

25. Zhou YJ, Li YY, Nie YQ, et al. Prevalence of fatty liver disease and its risk factors in the population of South China. World J Gastroenterol 2007; 13: 6419-6424.

26. Vanni E, Bugianesi E, Kotronen A, et al. From the metabolic syndrome to NAFLD or vice versa? Dig Liver Dis 2010; 42: 320330.

27. Alkassabany YM, Farghaly AG, El-Ghitany EM. Prevalence, risk factors, and predictors of nonalcoholic fatty liver disease among schoolchildren: a hospital-based study in Alexandria, Egypt. Arab J Gastroenterol 2014; 15: 76-81.

28. Wang Y, Zeng Y, Lin C, Chen Z, et al. Hypertension and non-alcoholic fatty liver disease proven by transient elastography. Hepatol Res 2016; 46: 1304-1310.

29. Oh S, Shida T, Yamagishi K, et al. Moderate to vigorous physical activity volume is an important factor for managing nonalcoholic fatty liver disease: A retrospective study. Hepatology 2015; 61: 1205-1215.

30. Santos AC, Ebrahim S, Barros H. Gender, socio-economic status and metabolic syndrome in middle-aged and old adults. BMC Public Health 2008; 8: 62.

31. Manuck SB, Phillips JE, Gianaros PJ, et al. Subjective socioeconomic status and presence of the metabolic syndrome in midlife community volunteers. Psychosom Med 2010; 72: 35-45. 